\title{
«PRINCIPE DE ANGLONA 5 Y 7»
}

\section{EDIFICIO DE NUEVA PLANTA}

Félix Echávarri Zarra, Arquitecto
El número 5 de la calle Principe de Anglona corresponde a un solar situado entre la medianeria del número 3 de la citada calle y la fachada lateral del edificio número 7 .

Dicho solar se ha contemplado por sus actuales propietarios dentro de una operación de conjunto que comprende la rehabilitación del Palacio de Anglona, con su jardín, y la del edificio del número 7 de la calle Principe de Anglona, asi como la construcción de un edificio de nueva planta sobre el solar que nos ocupa.

Si de hecho toda actuación en el casco antiguo supone un fuerte compromiso en cuanto a la elección del camino a seguir para integrar nuestra intervención dentro del entorno que nos corresponda, en este caso concreto, el compromiso se acentúa de forma especial, por la razón de que no estamos actuando en solitario, sino que trabajamos dentro de toda la operación de rehabilitación puesta en marcha a nuestro alrededor.

Observando el alzado completo de la calle en la que se encuentra nuestro proyecto, el primer problema a resolver es el de la composición volumétrica del conjunto que, como se puede ver, está fuertemente condicionada por el edificio existente, el número 3 , asi como por la necesidad de dejar libre el lateral del edificio del número 7 que es en realidad una fachada más del mismo, ya que abren todos sus huecos sobre nuestro solar; a partir de esta premisa se ha adoptado una composición que consideramos la más lógica, consistente en complementar las lineas definidas por el edificio medianero, con vistas a lograr un volumen único y armónico con el conjunto de los dos edificios $y$, de otra parte, en liberar una franja de terreno junto al número 7 que a la vez que resuelve el problema da la servidumbre de vistas de dicho edificio y nos sirve, a su vez, para dar solución de continuidad a las distintas alturas de cornisa y distintas proporciones de elementos que tienen los edificios existentes, uno a cada extremo de la calle.

Una vez tomada esta decisión, hemos realizado nuestro proyecto, respetando todas las lineas generales de la fachada del edificio al cual nos adosamos, estableciendo la continuidad minuciosa de impostas, cornisa y cumbrera con elementos horizontales claramente definitorios de la fachada y de la volumetría general. Asimismo, y dentro de la decisión de armonizar el conjunto, se ha proyectado la fachada con los mismos esquemas de elementos básicos del edificio existente, una vez analizados las proporciones y materiales de los mismos, como son: huecos y recercados, elementos de cerrajeria y paños ciegos, poniendo especial atención en dotar a todos ellos de un diseño propio, huyendo de un mimetismo que resultaria en exceso peligroso.

En cuanto a la composición interna del edificio, hay que resaltar como punto singular del mismo, el diseño de la zona interior libre de solar como un jardin, con voluntad de dotar al mismo de una fuerte personalidad, convirtiéndolo en un lugar de estancia y disfrute visual para los usuarios de las viviendas.

Se han proyectado una planta de sótano y una de semisótano, destinadas ambas a garaje aparcamiento. La planta baja consta, además, de varios locales con uso presumible de despachos, con los elementos comunes de portal, arranque de escaleras y ascensor y un garaje que conecta todo lo anterior con el jardin interior; se ha puesto especial énfasis en lograr con todos estos espacios un eje longitudinal que ya desde la entrada del edificio nos introduzca visualmente con el jardín interior. Baste añadir que sobre la planta baja se desarrollan cinco plantas en línea de fachada destinadas a viviendas, siendo la última planta abuhardillada, siguiendo la pendiente de la cubierta con viviendas en dos alturas conectadas ambas en un espacio único. 


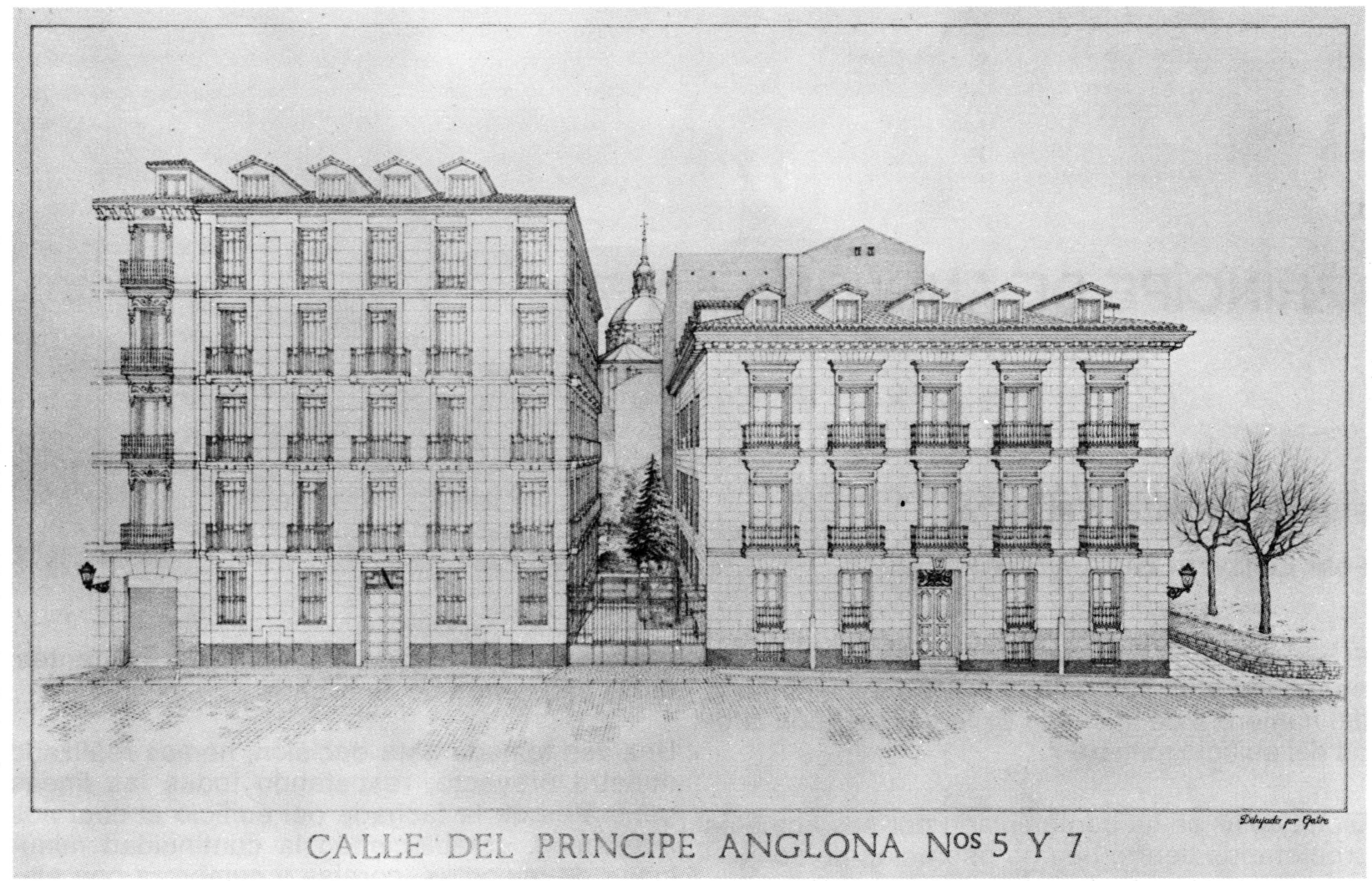

\section{REHABILITACION DEL INMUEBLE}

Ignacio Blanco Lecroisey, Arquitecto

$128-19$

El Plan Especial de Protección y Conservación de edificios y conjuntos históricos y artísticos de la Villa de Madrid, al ser un documento que se alinea claramente por la conservación, protección y defensa del patrimonio urbano, cambia el enfoque estrictamente historicista, por una actitud más culta de revalorización de la ciudad existente promoviendo su rehabilitación.

El edificio que nos ocupa, sin ser una pieza especialmente singular de la arquitectura madrileña de principios de siglo; forma un hecho irrepetible de gran dignidad y porte. Su aspecto es noble, de buena proporcionalidad entre su longitud y altura, así como de una adecuada armonia entre sus huecos y buhardillas. Las posibilidades de rehabilitación, a la hora de proyectar, no ofrecian tantas preocupaciones como su vecino el Palacio de Anglona, donde las distancias entre huecos creaban problemas de sobredimensionamiento de viviendas.

Situado en el viejo Madrid y en una zona tranquila que gira alrededor de la Plaza de la Paja, contiene una gran dosis de casticismo. La intervención apuntaba más al respeto al edificio que a las soluciones geniales.
Circunstancias de indolencia, ya conocidas, habian dejado al edificio en una situación de abandono, pero todavía en tal estado que era posible su recuperación... tanto en sus fachadas como en su interior.

El edificio se encontraba ahora con un grupo de personas con diferentes cometidos, que creian en él y lo querian preservar de la ruina, volviéndolo a incluir en el tejido urbano. Esto se iba a conseguir mediante la restauración y conservación de lo externo y su rehabilitación en lo interno, sin cambio de usos pero adecuándolo, en el tamaño de sus viviendas, más pequeñas, de tal forma que puedan ser adquiridas y mantenidas por nuevos vecinos, que de esta manera, evitan tener que salir de su ciudad.

En este sentido, la Teoria de la Rehabilitación se hace un constante aprendizaje, un continuo tejer y destejer en el que el intercambio de criterios y soluciones es constante: lo que es bueno ahora, no lo es dentro de un mes. La ecuación, de muchas variables que encierra cualquier proceso rehabilitador, no tiene una solución, sino varias, y éstas a su vez van cambiando con el tiempo.

El Arquitecto, junto con el Promotor, van descubriendo soluciones que hay que adecuar constantemente al mercado, a la evolución de nuestros propios criterios y a la rasante que cualquier economia pone a sus productos. Por otra parte, está el edificio con su propia limitación y con la 
protección que queremos estimular, los proyectos van evolucionando en una larga y controvertida partida. De un edificio que se proyecta básicamente con 18 viviendas, se cambia en ejecución a 16 y se termina en 12 .

Los reflejos para captar cualquier solución innovadora tienen que estar a punto. No vale aburrirse y la experiencia de otras actuaciones sólo vale para comprobar que aqui no hay experiencia que valga. Las recetas están prohibidas.

Las obras de Príncipe de Anglona, 7 han sido las necesarias para la adecuación, mejora de habitabilidad y redistribución de espacio interior, al que me he referido, manteniendo en cualquier caso las caracteristicas estructurales del edificio, aparte de coriservar, restaurar y consolidar el mismo. Las soluciones en la planta de cubiertas, que hemos tenido que realizar totalmente de nuevo, debido a su mal estado, han adoptado unas tecnologias muy sencillas y económicas. El conjunto de la obra se ha realizado en un tiempo de diez meses aproximadamente y se ha recurrido a contratas artesanales sin complicaciones de infraestructura empresarial. Para la sincronización de oficios y de la obra en general se ha huido de las empresas importantes y se ha tenido una gran docilidad en la organización. Los materiales más expresivos se han recuperado en su totalidad, desde las ventanas, incluidos todos sus herrajes, hasta la tarima que se ha reciclado para su utilización... Escalera, barandillas, peldaños, balcones... y un largo etc, no han entrado en la obra por la sencilla razón de que no han salido. Los muros se han consolidado donde ha hecho falta y la estructura de cubierta apoya sobre sus muros sin necesidad de nuevos apósitos. Se ha aislado con rigor y se ha calefactado una a una todas las viviendas para mayor independencia de los usuarios. Las contraventanas de madera se han limpiado una a una en el patio de la casa, se han encerado y vuelto a colocar. Las cocinas y cuartos de baño se han resuelto sin ningún tipo de materiales lujosos, pero entendemos que son muy dignos y bien dotados. Creemos que hemos oído mucho a nuestros clientes y hemos mantenido a lo largo de la obra una economia de crisis de la que estamos orgullosos.

\section{Equipo de Trabajo que ha intervenido en la rehabilitación del Palacio de Anglona}

Dirección-Coordinación:

Fernando Moreno de Barreda.

Arquitectos:

Ignacio Blanco Lecroisey.

Jaime Martinez de Ubago.

Carlos Boyer Monsalve.

Aparejadores:

Fernando Berrio Zazo.

José Ayala Blas.

Juan Jesús Carretero Vera.

Paisajista:

Lucia Serredi.

Restauración de Pinturas:

Antonio Sánchez Barriga.

Fernando Llorente Sagaseta.

Karen Lee Otera.
Teresa Fernández Muro. Judith Sáenz de Tejada.

Restauración de otros elementos: Carlos Vicente.

Segundino González Arenes.

Estudios y colaboraciones: Antonio Bonet Correa. Virginia Tovar. Juan José Luna. Maria José Arnáiz Gorroño. Enrique Gutièrrez de Calderón.

Documentación Histórica: Paloma Diaz Martinez Esparza. José Luis Sancho. 